\title{
More dependent types for distributed arrays
}

\author{
Wouter Swierstra
}

Published online: 8 September 2011

(C) The Author(s) 2011. This article is published with open access at Springerlink.com

\begin{abstract}
Locality-aware algorithms over distributed arrays can be very difficult to write. Yet such algorithms are becoming more and more important as desktop machines boast more and more processors. This paper shows how a dependently-typed programming language can aid in the development of these algorithms and statically ensure that every well-typed program will only ever access local data. Such static guarantees can help catch programming errors early on in the development cycle and maximise the potential speedup that multicore machines offer. At the same time, the functional specification of effects presented here facilitates the testing of and reasoning about algorithms that manipulate distributed arrays.
\end{abstract}

Keywords Dependent types $\cdot$ Agda $\cdot$ Distributed arrays $\cdot$ Type systems $\cdot$ Domain specific languages

\section{Introduction}

Computer processors are not becoming significantly faster. To satisfy the demand for more and more computational power, manufacturers are now assembling computers with multiple microprocessors. It is hard to exaggerate the impact this will have on software development: tomorrow's programming languages must embrace parallel programming on multicore machines.

Researchers have proposed several new languages to maximise the potential performance gain that multicore processors offer $[1,6-8,12,21]$. Although all these languages are different, they share the central notion of a distributed array, where the elements of an array may be distributed over separate processors or even over separate machines. To write efficient code, programmers must ensure that processors only access local parts of a distributed array-it is much faster to access data stored locally than remote data on another core.

When writing such locality-aware algorithms it is all too easy to make subtle mistakes. Programming languages designed specifically for distributed computing, such as X10 [8],

W. Swierstra ( $₫)$

Vector Fabrics, Paradijslaan 28, Eindhoven, The Netherlands

e-mail: wouter@vectorfabrics.com 
require all arrays operations to be local. Any attempt to access non-local data results in an exception. To preclude such errors, X10's type system guarantees that programs only access local parts of a distributed array [9, 17]. The proposed type system is fairly intricate and consists of a substantial number of type rules that keep track of locality information. Proving meta-theoretical results about such a system, such as the decidability of type checking, is no trivial result.

This paper explores an alternative avenue of research. Designing and implementing a type system from scratch is a lot of work. New type systems typically require extensive proofs of various meta-theoretical results. Instead, this paper shows how to tailor a powerful type system to enforce certain properties-resulting in a domain-specific embedded type system. This type system immediately inherits all the desirable properties of our dependentlytyped host type system, such as subject reduction, decidable type checking, and principle typing. Functional programmers have studied domain-specific embedded languages for years [11]; the time is ripe to take these ideas one step further.

Previous work described a pure specification of several parts of the IO monad [23], the interface between pure functional languages such as Haskell [19] and the 'real world.' By providing functional, executable specifications you can test, debug, and reason about impure programs as if they were pure. When you release the final version of your code, you can replace these pure specifications with their impure, more efficient, counterparts. In the presence of dependent types, these specifications can provide even stronger static guarantees about our programs. To this end, this paper makes several contributions:

- We will begin by giving a pure specification of arrays (Sect. 3). This specification is total: there is no way to access unallocated memory; there are no 'array index out of bounds' exceptions. As a result, these specifications can not only be used to program with, but also facilitate formal proofs about array algorithms. To make this specification usable, we must overcome a problem related to the weakening of address locations.

- Distributed arrays pose more of a challenge. Section 4 shows how to enforce locality constraints, while still providing programmers with place-shifting operators. The pure specification is, once again, executable and total: it can be interpreted both as a domain-specific embedded language for writing algorithms on distributed arrays and as an executable denotational model for specifying and proving properties of such algorithms.

- Finally, we will see how programmers may write their own locality-aware control structures (Sect. 4.3), how to implement simple distributed algorithms using these control structures (Sect. 4.4), and how to define combinators that describe data distributions (Sect. 4.5). We will conclude by discussing further work and the limitations of this approach (Sect. 5).

Throughout this paper, I will use the dependently-typed programming language Agda [16] as a vehicle of explanation. In fact, using lhs2TeX [13], the sources of this paper generate an Agda program that can be compiled and executed. In the coming section, I will briefly introduce the syntax of Agda by means of several examples, as it may be unfamiliar to many readers.

\section{An overview of Agda}

Data types in Agda can be defined using a similar syntax to that for Generalized Algebraic Data Types, or GADTs, in Haskell [20]. For example, consider the following definition of the natural numbers. 


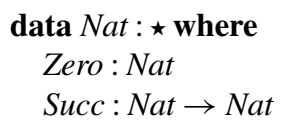

There is one important difference with Haskell. We must explicitly state the kind of the data type that we are introducing; in particular, the declaration Nat: $\star$ states that Nat is a base type.

We can define functions by pattern matching and recursion, just as in any other functional language. To define addition of natural numbers, for instance, we could write:

$$
\begin{aligned}
& +_{-}: \text {Nat } \rightarrow \text { Nat } \rightarrow \text { Nat } \\
& \text { Zero }+m=m \\
& \text { Succ } n+m=\operatorname{Succ}(n+m)
\end{aligned}
$$

Note that Agda uses underscores to denote the positions of arguments when defining new operators.

Polymorphic lists are slightly more interesting than natural numbers:

$$
\begin{aligned}
& \text { data List }(a: \star): \star \text { where } \\
& \text { Nil }: \text { List } a \\
& \text { Cons }: a \rightarrow \text { List } a \rightarrow \text { List } a
\end{aligned}
$$

To uniformly parameterise a data type, we can write additional arguments to the left of the colon. In this case, we add $(a: \star)$ to our data type declaration to state that lists are type constructors, parameterised over a type variable $a$ of kind $\star$.

Just as we defined addition for natural numbers, we can define an operator that appends one list to another:

$$
\begin{aligned}
& \text { append }:(a: \star) \rightarrow \text { List } a \rightarrow \text { List } a \rightarrow \text { List a } \\
& \text { append a Nil ys }=y s \\
& \text { append } a(\text { Cons } x \times s) \text { ys }=\text { Cons } x(\text { append a xs ys })
\end{aligned}
$$

The append function is polymorphic. In Agda, such polymorphism can be introduced via the dependent function space, written $(x: a) \rightarrow y$, where the variable $x$ may occur in the type $y$. This particular example of the dependent function space is not terribly interesting: it corresponds to parametric polymorphism. Later we will encounter more interesting examples, where types depend on values.

One drawback of using the dependent function space for such parametric polymorphism, is that we must explicitly instantiate polymorphic functions. For example, the recursive call to append in the Cons case takes a type as its first argument. Fortunately, Agda allows us to mark certain arguments as implicit. Using implicit arguments, we could also define append as in any other functional language:

$$
\begin{aligned}
& \text { append }:\{a: \star\} \rightarrow \text { List } a \rightarrow \text { List } a \rightarrow \text { List a } \\
& \text { append Nil ys }=y s \\
& \text { append }(\text { Cons } x x s) \text { ys }=\text { Cons } x(\text { append } x s y s)
\end{aligned}
$$

Arguments enclosed in curly brackets, such as $\{a: \star\}$, are implicit: we do not write $a$ to the left of the equals sign and do not pass a type argument when we make a recursive call. The Agda type checker will automatically instantiate this function whenever we call it, much 
in the same way as type variables are automatically instantiated in Haskell. By enclosing a function's argument in curly brackets, we can explicitly instantiate or pattern match on an implicit argument.

Besides polymorphic data types, Agda also supports indexed families, a dependently typed analogue of Haskell's GADTs. Indexed families, however, are more general as they also capture data types that are indexed by values instead of types. For example, we can define the family of finite types:

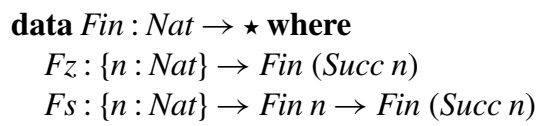

The type Fin $n$ corresponds to a finite type with $n$ distinct values. For example, Fin 1 is isomorphic to the unit type; Fin 2 is isomorphic to Bool. Note that the argument $n$ is left implicit in both the constructors of Fin. From the types of these constructors, it is easy to see that Fin 0 is uninhabited. For every $n$, the Fs constructor embeds Fin $n$ into Fin (Succ n); the $F z$ constructor, on the other hand, adds a single new element to Fin (Succ n) that was not in Fin $n$. This inductive argument shows that Fin $n$ does indeed have $n$ elements.

Agda has many other features, such as records and a module system, that we will hardly use in this paper. Although there are a few more concepts we will need, we will discuss them as they pop up in later sections.

\section{Mutable arrays}

With this brief Agda tutorial under our belt, we can start our specification of mutable arrays. We will specify three different operations on arrays: the creation of new arrays; reading from an array; and updating a value stored in an array. Before we can define the behaviour of these operations, we need to introduce several data types to describe the layout and contents of memory. Using these data types, we can proceed by defining an $I O$ type that captures the syntax of array operations. To program with these operators, we need to resolve a few technical problems. Finally, we will define a run function that describes how the array operations affect the heap, assigning semantics to our syntax. This semantics can be used to simulate and reason about computations on mutable arrays in a pure functional language. When compiled, however, these operations should be replaced by their more efficient, low-level counterparts.

To keep things simple, we will only work with flat arrays storing natural numbers. This is, of course, a drastic oversimplification. The techniques we present here, however, can be adapted to cover multidimensional arrays that may store different types of data using a universe construction, as is done in my $\mathrm{PhD}$ thesis [22].

To avoid confusion between numbers denoting the size of an array and the data stored in an array, we introduce the Data type synonym. Throughout the rest of this paper, we will use Data to refer to the data stored in arrays; the Nat type will always refer to the size of an array.

\section{Data: *}

Data $=$ Nat

Using the Fin type, we can give a functional specification of arrays of a fixed size by mapping every index to the corresponding value. 
Array: Nat $\rightarrow \star$

Array $n=$ Fin $n \rightarrow$ Data

How should we represent the heap? We need to be a bit careful—as the heap will store arrays of different sizes its type should explicitly state how many arrays it stores and how large each array is. To accomplish this, we begin by introducing a data type representing the shape of the heap:

$$
\begin{aligned}
& \text { Shape }: \star \\
& \text { Shape }=\text { List Nat }
\end{aligned}
$$

The Shape of the heap is a list of natural numbers, representing the size of the arrays stored in memory.

We can now define a Heap data type that is indexed by a Shape. The Empty constructor corresponds to an empty heap; the Alloc constructor adds an array of size $n$ to any heap of shape $n s$ to build a larger heap with the layout Cons $n n s$.

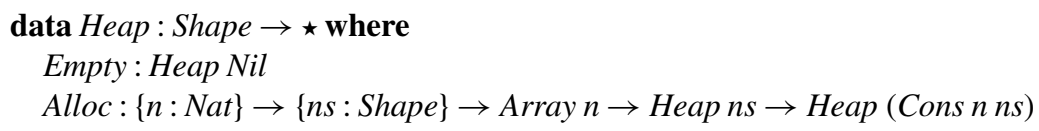

Finally, we will want to model references, denoting locations in the heap. A value of type Loc $n n s$ corresponds to a reference to an array of size $n$ in a heap with shape $n s$. The Loc data type shares a great deal of structure with the Fin type. Every non-empty heap has a Top reference; any existing reference can be modified to denote the same location in a larger heap using the Pop constructor.

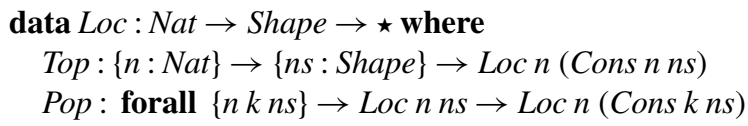

Note that in the type signature of the Pop constructor, we omit the types of three implicit arguments and quantify over them using the forall keyword. When we use the forall-notation, the types of $n, k$, and $n s$ are inferred from the rest of the signature by the Agda type checker. Alternatively, we could also have written the more verbose:

$$
\begin{aligned}
\text { Pop }:\{n: \text { Nat }\} & \rightarrow\{k: \text { Nat }\} \rightarrow\{n s: \text { Shape }\} \\
& \rightarrow \text { Loc } n n s \rightarrow \text { Loc } n(\text { Cons } k n s)
\end{aligned}
$$

We will occasionally use the forall-notation to make large type signatures somewhat more legible.

With these data types in place, we can define a data type capturing the syntax of the permissible operations on arrays. Crucially, the $I O$ type is indexed by two shapes: a value of type IO a ns ms denotes a computation that takes a heap of shape $n s$ to a heap of shape $m s$ and returns a result of type $a$. This pattern of indexing operations by an initial and final 'state' is a common pattern in dependently-typed programming [14]. 


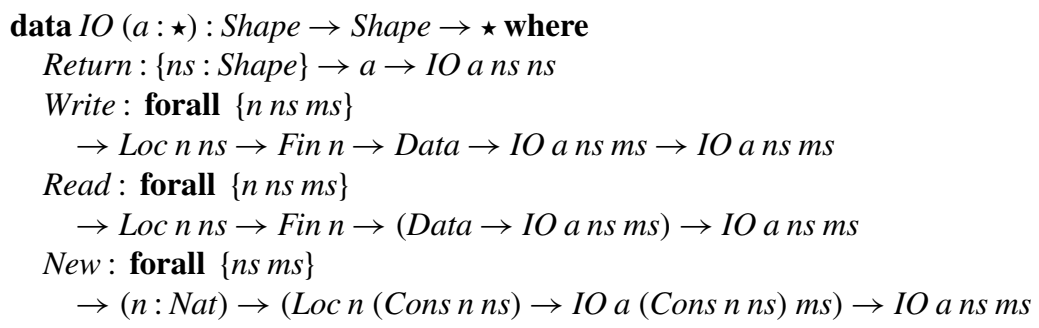

The $I O$ type has four constructors. The Return constructor returns a pure value of type $a$ without modifying the heap. The Write constructor takes four arguments: the location of an array of size $n$; an index in that array; the value to write at that index; and the rest of the computation. Similarly, reading from an array requires a reference to an array and an index. Instead of requiring the data to be written, however, the last argument of the Read constructor may refer to data that has been read. Finally, the New constructor actually changes the size of the heap. Given a number $n$, it allocates an array of size $n$ on the heap; the second argument of New may then use this fresh reference to continue the computation in a larger heap.

The $I O$ data type is a parameterised monad [3] - that is, a monad with return and bind operators that satisfy certain coherence conditions with respect to the Shape indices.

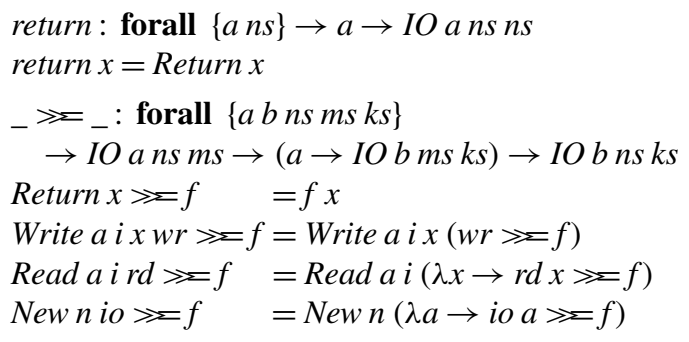

The return of the $I O$ data type lifts a pure value into a computation that can run on a heap of any size. Furthermore, return does not modify the shape of the heap. The bind operator, $\gg$, can be used to compose monadic computations. To sequence two computations, the heap resulting from the first computation must be a suitable starting point for the second computation. This condition is enforced by the type of the bind operator.

To facilitate programming using these array operations, we could define the functions readArray and newArray as follows:

$$
\begin{aligned}
& \text { readArray: forall }\{n n s\} \rightarrow \text { Loc } n n s \rightarrow \text { Fin } n \rightarrow \text { IO Data } n s n s \\
& \text { readArray a } i=\text { Read a i Return } \\
& n \text { newArray: forall }\{n s\} \rightarrow(n: N a t) \rightarrow I O(\text { Loc } n(\text { Cons } n n s)) n s(\text { Cons } n n s) \\
& n e w A r r a y n=N e w n \text { Return }
\end{aligned}
$$

There is a slight problem with these definitions. As we allocate new memory, the size of the heap changes; correspondingly, we must explicitly modify any existing pointers to denote locations in a larger heap. This problem is best illustrated with an example.

Consider the following sequence of array manipulations:

$$
\begin{aligned}
& \text { newArray } 4 \gg \lambda \text { array } 1 \\
& \quad \rightarrow \text { newArray } 8 \gg \lambda \text { array } 2 \\
& \quad \rightarrow \text { readArray array } 1 \mathrm{Fz}
\end{aligned}
$$


This allocates two arrays, before performing reading an index from the first array. The first array, stored at arrayl, has type Loc 4 (Cons $4 \mathrm{Nil}$ ); the second array has type Loc 8 (Cons 8 (Cons $4 \mathrm{Nil}$ )). When we want to read from the first array after the second allocation, the type checker expects an array location in a heap of shape (Cons 8 (Cons $4 \mathrm{Nil})$ ), and not Cons 4 Nil. Fortunately, adding a Pop constructor around arrayl yields a type correct program:

$$
\begin{aligned}
& \text { newArray } 4 \gg \lambda \text { array }_{1} \\
& \quad \rightarrow \text { newArray } 8 \gg \text { array }_{2} \\
& \quad \rightarrow \text { readArray }_{\left(\text {Pop } \text { array }_{1}\right) \mathrm{Fz}}
\end{aligned}
$$

We will refer to this as weakening the reference $a_{r r a y}$, in line with the usual terminology used for the weakening rules present in many logics.

Adding these extra Pop constructors by hand is tiresome and error-prone. In the coming sections, we will see how this process can be automated to some degree.

\subsection{Manual weakening}

Before we revisit our smart constructors, we need to develop a bit of machinery. We need to decide how many Pop constructors are necessary to weaken a location to denote a position in a larger heap. Put differently, we need to prove that one shape is a suffix of a larger shape. One way to represent such a proof is using an inductive data type:

$$
\begin{aligned}
& \text { data IsSuffix: Shape } \rightarrow \text { Shape } \rightarrow \star \text { where } \\
& \text { Base: forall }\{n s\} \rightarrow \text { IsSuffix ns ns } \\
& \text { Step: forall }\{m n s m s\} \rightarrow I s \text { Suffix } n s m s \rightarrow \text { IsSuffix ns (Cons } m \text { ms) }
\end{aligned}
$$

Every proof that $n s$ is a suffix of $m s$ is built from two constructors Base and Step. The base case corresponds to stating that every list is a suffix of itself; the step case states that if $n s$ is a suffix of $m s$ then $n s$ is also a suffix of Cons $m m s$ for any natural number $m$.

If we have such a proof that $n s$ is a suffix of $m s$, we can weaken any location of type Loc $n n s$ to make a new location of type Loc $n \mathrm{~ms}$. The weaken function does exactly this:

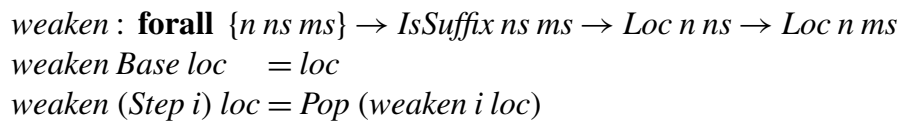

It proceeds by induction over the proof argument, adding a Pop constructor for every step. While the weaken function adds the necessary Pop constructors, there is still no way to compute its proof argument automatically. There is an alternative representation of such proofs that does facilitate such automation.

\subsection{An alternative proof}

In type theory, one way to formulate the proposition that two values are equal is by constructing an inhabitant of the following type:

$$
\begin{aligned}
& \text { data }_{-} \equiv_{-}\{a: \star\}(x: a): a \rightarrow \star \text { where } \\
& \quad \text { Refl }_{x} \equiv x
\end{aligned}
$$


That is, the only canonical proof that two objects are equal is by reflexivity. This type plays a fundamental role in intensional type theory [15].

Whenever we pattern match on an equality proof, we learn how two values are related. For example, suppose we want to write the following function:

$$
\text { subst }:\{a: \star\} \rightarrow(P: a \rightarrow \star) \rightarrow(x: a) \rightarrow(y: a) \rightarrow x \equiv y \rightarrow P x \rightarrow P y
$$

What patterns should we write on the left-hand side of the definition? Clearly, any argument of type $x \equiv y$ must be Refl. As soon as we match on that argument, however, we learn something about $x$ and $y$, i.e., they must be the same. We will write this as follows:

$$
\text { subst Px } x \text { Refl } p x=\cdots
$$

The pattern $x$ means 'the value of this argument can only be equal to $x$.' Such 'dot-patterns' appear naturally once you start programming with data types indexed by values.

We can decide whenever two shapes are equal, that is, we can define a function with the following type:

$$
\text { decEqShape }:(s: \text { Shape }) \rightarrow(t: \text { Shape }) \rightarrow \text { Either }(s \equiv t)((s \equiv t) \rightarrow \perp)
$$

Here Either denotes the disjoint sum of two types and $\perp$ is the empty type. The definition of decEqShape is fairly unremarkable. We traverse the lists $s$ and $t$, comparing every element. If the elements of both lists are equal at every position, the entire lists are equal; if the lists have different lengths or store different elements, the two shapes are distinct.

Using this function, we can define the following function that checks whether or not one list is a suffix of another:

$$
\begin{aligned}
& \leq_{-}: \text {Shape } \rightarrow \text { Shape } \rightarrow \text { Bool } \\
& \text { Nil } \leq s \quad=\text { True } \\
& \text { Cons u } s \leq \text { Nil = False } \\
& \text { Cons } u s \leq \text { Cons } v t \text { with decEqShape (Cons u s) (Cons v } t \text { ) } \\
& \text { Cons } u s \leq \text { Cons .u } . s \mid \text { Inl Refl = True } \\
& \text { Cons } u s \leq \text { Cons } v t \mid \text { Inr } q=(\text { Cons } u s) \leq t
\end{aligned}
$$

Here we use a with clause, a local pattern match similar to Haskell's case expressions. In the inductive step, we make a call to the auxiliary function $d e c E q S h a p e$. On the next lines we repeat the left-hand side of the function definition, followed by the new patterns Inl Refl and In $q$. We separate the new patterns introduced by the with clause from the original function arguments by a vertical bar. If we learn that the two lists are equal, it follows that the first list is a suffix of the second. Otherwise, we continue with a recursive call.

Now we can reflect any Bool into $\star$ as follows:

$$
\begin{aligned}
& \text { So }: \text { Bool } \rightarrow \text { } \\
& \text { So True }=() \\
& \text { So False }=\perp
\end{aligned}
$$

Using this definition, we give an alternative definition of the IsSuffix predicate: a shape $s$ is a suffix of a shape $t$ if and only if $S o(s \leq t)$ is inhabited. This alternative definition is equivalent to the definition using IsSuffix. Proving the equivalence in one direction corresponds to defining a function with the following type: 


$$
\text { equiv }:(s: \text { Shape }) \rightarrow(t: \text { Shape }) \rightarrow \text { So }(s \leq t) \rightarrow I s \text { Suffix } s t
$$

The definition is reasonably straightforward: we pattern match on $s$ and $t$, using the proof argument to kill off any impossible branches.

So why go through all this effort to write an equivalent representation of the inductive IsSuffix data type? The two forms of proof are useful for different reasons:

Is Suffix s $t$ By defining the proof as an inductive data type, we can pattern match on proofs. We use this to define the weaken function. Unfortunately, we need to write an inhabitant of IsSuffix by hand to pass to the weaken function.

So $(s \leq t)$ By defining the $\leq$-operator we have written a function that decides when one list is a suffix of another. In particular, for any pair of closed shapes $s \leq t$ reduces to either True or False. Correspondingly, the type $S o(s \leq t)$ is either trivial or uninhabited.

The central idea behind our smart constructors is to weaken using the inductive representation, but to require a trivial implicit witness.

\subsection{Smart constructors}

With this in mind, we revise our smart constructors for reading from and writing to references. These smart constructors now require an implicit proof that $s$ is a suffix of $t$. Using our equiv function, we can compute the inductive representation of such a proof. Using this inductive representation, we can weaken locations as necessary.

$$
\begin{aligned}
& \text { readArray: forall }\{n n s m s\} \rightarrow\{p: \text { So }(n s \leq m s)\} \\
& \quad \rightarrow \text { Loc } n n s \rightarrow \text { Fin } n \rightarrow I O \text { Data } m s m s \\
& \text { readArray }\{n\}\{n s\}\{m s\}\{p\} \text { a } i=\text { Read (weaken (equiv ns ms } p) \text { a) i Return }
\end{aligned}
$$

The beauty of this solution is that Agda will automatically instantiate implicit arguments of type (). In other words, for any closed $I O$ term a programmer need not worry about passing proof arguments. For example, we can now write:

$$
\begin{aligned}
& f: \text { IO Nat Nil }(\text { Cons } 8(\text { Cons } 4 \mathrm{Nil})) \\
& \begin{aligned}
f= & \text { newArray } 4 \gg \text { array }_{1} \\
& \rightarrow \text { newArray } 8 \gg \text { array }_{2} \\
& \rightarrow \text { readArray array }_{1} \mathrm{Fz}
\end{aligned}
\end{aligned}
$$

The smart constructors will now automatically weaken $\operatorname{array}_{1}$ after $\operatorname{array}_{2}$ has been allocated.

Unfortunately, not all in the garden is rosy. If we have an open term, Agda will warn you that it cannot find a suitable proof argument. For example, consider the following function that reads the first and only location of any array storing a single element:

readOne $:\{n s:$ Shape $\} \rightarrow$ Loc $1 n s \rightarrow I O$ Nat ns ns

readOne $a=$ readArray $a \mathrm{Fz}$

While any particular call to readOne is safe, Agda fails to automatically deduce that $S o$ ( $n s \leq$ $n s$ ) reduces to the unit type. Even though it is clearly true for any particular choice of $n s$ and we can fill in the proof argument manually, the techniques we have outlined here fail to provide the required proof automatically.

Without more language support it is unlikely that we can avoid this restriction. 


\subsection{Denotational model}

We have described the syntax of array computations using the $I O$ data type, but we have not specified how these computations behave. Recall that we can model arrays as functions from indices to natural numbers:

Array: Nat $\rightarrow \star$

Array $n=$ Fin $n \rightarrow$ Data

Before specifying the behaviour of $I O$ computations, we define several auxiliary functions to update an array and lookup a value stored in an array.

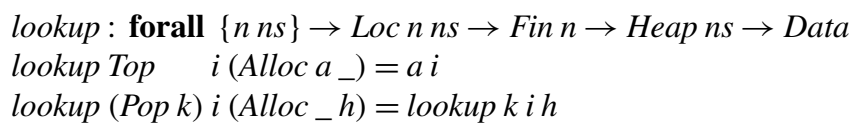

The lookup function takes a reference to an array $l$, an index $i$ in the array at location $l$, and a heap, and returns the value stored in the array at index $i$. It dereferences $l$, resulting in a function of type Fin $n \rightarrow$ Data; the value stored at index $i$ is the result of applying this function to $i$.

Next, we define a pair of functions to update the contents of an array.

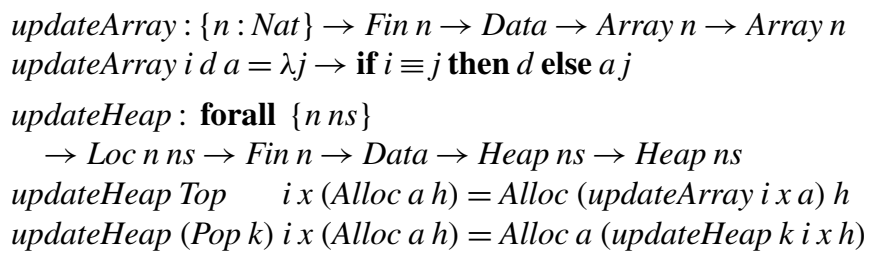

The updateArray function overwrites the data stored at a single index. The function updateHeap updates a single index of an array stored in the heap. It proceeds by dereferencing the location on the heap where the desired array is stored and updates it accordingly, leaving the rest of the heap unchanged.

We now have all the pieces in place to assign semantics to $I O$ computations. The run function below takes a computation of type $I O$ a $n s m s$ and an initial heap of shape $n s$ as arguments, and returns a pair consisting of the result of the computation and the final heap of shape $m s$.

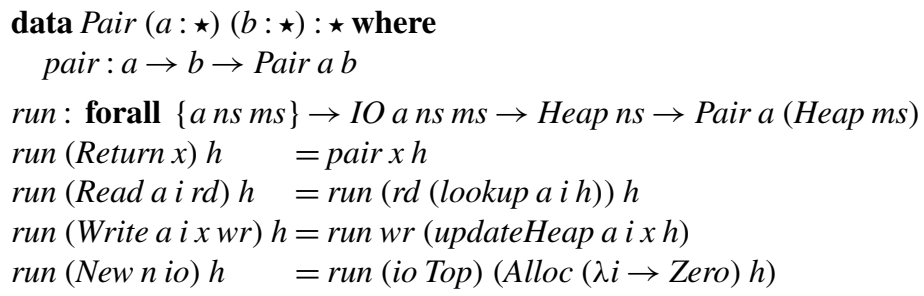

The Return constructor simply pairs the result and heap; in the Read case, we lookup the data from the heap and recurse with the same heap; for the Write constructor, we recurse with an appropriately modified heap; finally, when a new array is created, we extend the 
heap with a new array that stores Zero at every index, and continue recursively. Note that, by convention, the Top constructor always refers to the most recently created reference. Our smart constructors will add additional Pop constructors when new memory is allocated.

We refer to this specification as a denotational model. Agda is a programming language based on a consistent type theory. In a sense, the run function constitutes a denotational semantics of mutable arrays. By implementing these semantics in Agda, we build an executable denotational model in Agda's type theory.

\subsection{Example}

Using our smart constructors and the monad operators, we can now define functions that manipulate arrays. For example, the swap function exchanges the value stored at two indices:

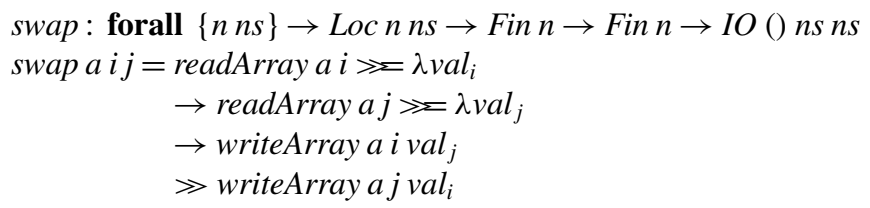

In a dependently-typed programming language such as Agda, we can prove properties of our code. For example, we may want to show that swapping the contents of any two array indices twice, leaves the heap intact :

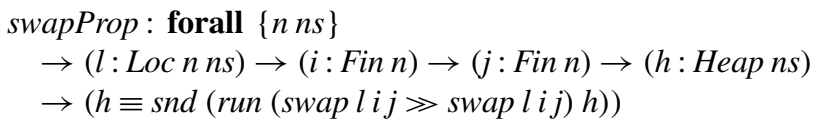

The proof requires a lemma about how updateHeap and lookupHeap interact and is not terribly interesting in itself. The fact that we can formalise such properties and have our proof verified by a computer is much more exciting.

\section{Distributed arrays}

Arrays are usually represented by a continuous block of memory. Distributed arrays, however, can be distributed over different places - where every place may correspond to a different core on a multiprocessor machine, a different machine on the same network, or any other configuration of interconnected computers.

We begin by determining the type of places, where data is stored and code is executed. Obviously, we do not want to fix the type of all possible places prematurely: you may want to execute the same program in different environments. Yet regardless of the exact number of places, there are certain operations you will always want to perform, such as iterating over all places, or checking when two places are equal.

We therefore choose to abstract over the number of places in the module we will define in the coming section. Agda allows modules to be parameterised:

\section{module DistrArray (placeCount : Nat) where}

When we import the DistrArray module, we are obliged to choose the number of places. Typically, there will be one place for every available processor. From this number, we can define a data type corresponding to the available places: 


\section{Place : $\star$ \\ Place $=$ Fin place Count}

The key idea underlying our model of locality-aware algorithms is to index computations by the place where they are executed. The new type declaration for the $I O$ monad corresponding to operations on distributed arrays will become:

$$
\text { data } D I O(a: \star): \text { Shape } \rightarrow \text { Place } \rightarrow \text { Shape } \rightarrow \star \text { where }
$$

You may want to think of a value of type DIO a ns $p m s$ as a computation that can be executed at place $p$ and will take a heap of shape $n s$ to a heap of shape $m s$, yielding a final value of type $a$.

We strive to ensure that any well-typed program written in the $D I O$ monad will never access data that is not local. The specification of distributed arrays now poses a twofold problem: we want to ensure that the array manipulations from the previous section are 'localityaware,' that is, we must somehow restrict the array indices that can be accessed from a certain place; furthermore, X10 facilitates several place-shifting operations that change the place where certain chunks of code are executed. As we shall see in the rest of this section, both these issues can be resolved quite naturally.

\subsection{Regions, points, and distributed arrays}

Before we define the $D I O$ monad, we need to introduce several new concepts. In what follows, we will try to stick closely to X10's terminology for distributed arrays. Every array is said to have a region associated with it. A region is a set of valid index points. A distribution specifies a place for every index point in a region.

Once again, we will only treat flat arrays storing natural numbers and defer any discussion about how to deal with more complicated data structures for the moment. In this simple case, a region merely determines the size of the array.

$$
\begin{aligned}
& \text { Region : } \star \\
& \text { Region }=N a t
\end{aligned}
$$

As we have seen in the previous section, we can model array indices using the Fin data type:

\section{Point : Region $\rightarrow \star$ \\ Point $n=$ Fin $n$}

To model distributed arrays, we now need to consider the distribution that specifies where this data is stored. In line with existing work [9], we assume the existence of a fixed distribution. Agda's postulate expression allows us to assume the existence of a distribution, without providing its definition.

\section{postulate}

$$
\text { distr: forall }\{n n s\} \rightarrow \text { Loc } n n s \rightarrow \text { Point } n \rightarrow \text { Place }
$$

We have implemented several of X10's combinators for defining distributions, which we will present shortly. 
Now that we have all the required auxiliary data types, we proceed by defining the DIO monad. As it is a bit more complex than the data types we have seen so far, we will discuss every constructor individually.

The Return constructor is analogous to one we have seen previously for the $I O$ monad: it lifts any pure value into the $D I O$ monad.

Return $:\{p:$ Place $\} \rightarrow\{n s:$ Shape $\} \rightarrow a \rightarrow$ DIO a ns $p$ ns

The Read and Write operations are more interesting. Although they correspond closely to the operations we have seen in the previous section, their type now keeps track of the place where they are executed. Any read or write operation to point $p t$ of an array $l$ can only be executed at the place specified by the distribution. This invariant is enforced by the types of our constructors:

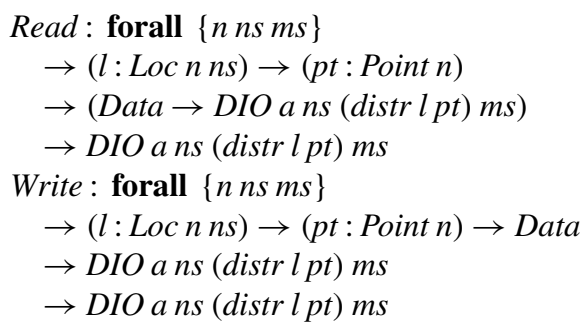

In contrast to Read and Write, new arrays can be allocated at any place.

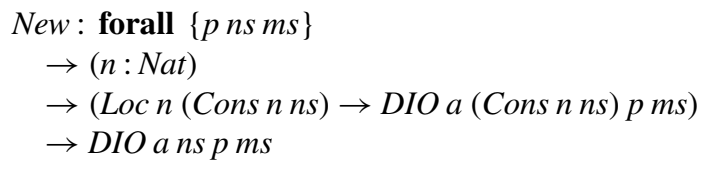

Finally, we add a constructor for a place-shifting operator. Using this At operator lets us execute a computation at another place.

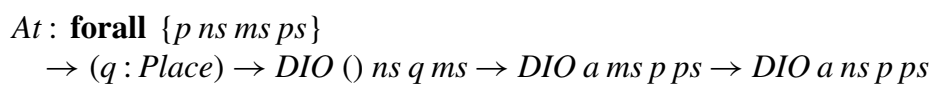

Note that we will discard the result of the computation that is executed at another place. We therefore require this computation to return an element of the unit type.

We can add our smart constructors for each these operations, as we have done in the previous section. We can also show that $D I O$ is indeed a parameterised monad. We have omitted the definitions of the return and bind operators for the sake of brevity:

$$
\begin{aligned}
& \text { return: forall }\{n s a p\} \rightarrow a \rightarrow \text { DIO a ns } p n s \\
& { }_{-}{ }_{-} \text {: forall }\{n s m s k s a b p\} \\
& \quad \rightarrow \text { DIO A } n s p m s \rightarrow(A \rightarrow D I O \text { B } m s p k s) \rightarrow \text { DIO B } n s p k s
\end{aligned}
$$

It is worth noting that the bind operator $\gg$ can only be used to sequence operations at the same place. 


\subsection{Denotational model}

To run a computation in the $D I O$ monad, we follow the run function defined in the previous section closely. Our new run function, however, must be locality-aware. Therefore, we parameterise the run function explicitly by the place where the computation is executed.

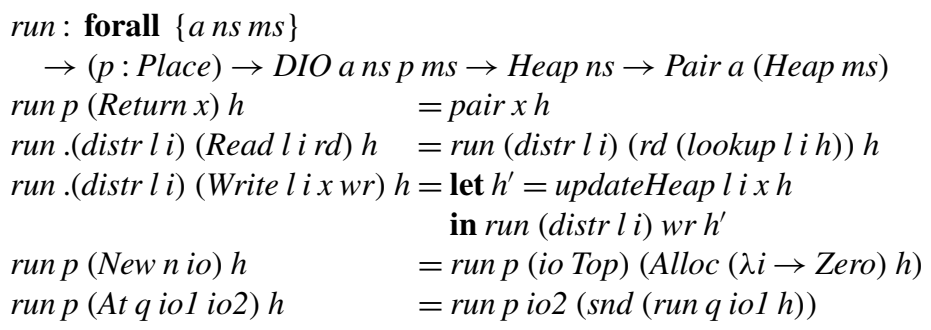

Now we can see that the Read and Write operations may not be executed at any place. Recall that the Read and Write constructors both return computations at the place distr $l i$. When we pattern match on a Read or Write, we know exactly what the place argument of the run function must be. Correspondingly, we do not pattern match on the place argument-we know that the place can only be distr $l i$, as is indicated by the dot-pattern.

The other difference with respect to the previous run function, is the new case for the At constructor. In that case, we sequence the two computations iol and io2. To do so, we first execute the iol at $q$, but discard its result; we continue executing the second computation io 2 with the heap resulting from the execution of iol at the location $p$. Conform to previous proposals [10], we have assumed that iol and io2 are performed synchronously-executing iol before continuing with the rest of the computation. Using techniques to model concurrency that we have presented previously [23], we believe we could give a more refined treatment of the X10's globally asynchronous/locally synchronous semantics and provide specifications for X10's clocks, finish, and force constructs.

\subsection{Locality-aware combinators}

Using the place-shifting operator at, we can define several locality-aware control structures. With our first-class distribution and definition of Place, we believe there is no need to define more primitive operations.

The distributed map, for example, applies a function to all the elements of a distributed array at the place where they are stored. We define it in terms of an auxiliary function, for, that iterates over all the indices of an array:

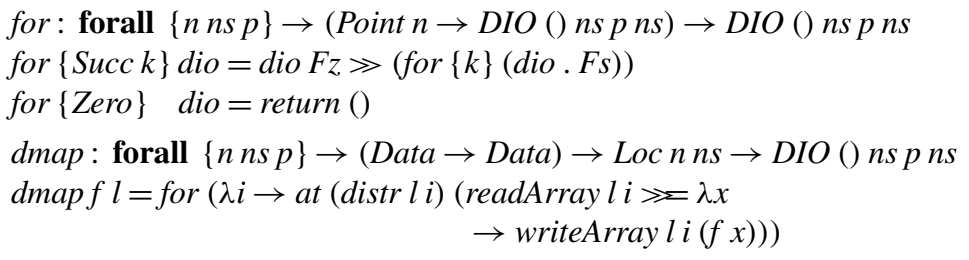

Besides dmap, we implement two other combinators: forallplaces and ateach. The forallplaces operation executes its argument computation at all available places. We define it using the for function to iterate over all places. The ateach function, on the other hand, 
is a generalisation of the distributed map operation. It iterates over an array, executing its argument operation once for every index of the array, at the place where that index is stored.

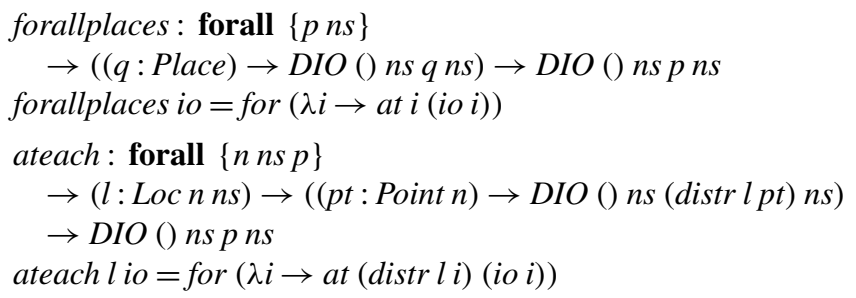

\subsection{Example}

We will now show how to write a simple algorithm that sums all the elements of a distributed array. To do so efficiently, we first locally sum all the values at every place. To compute the total sum of all the elements of the array, we add together all these local sums. In what follows, we will need the following auxiliary function, increment:

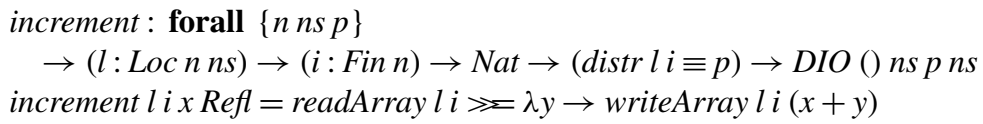

Note that increment is a bit more general than strictly necessary. We could return a computation at distr $l i$, but instead we choose to be a little more general: increment can be executed at any place, as long as we have a proof that this place is equal to distr $l i$. The $\equiv-$ type is inhabited by single constructor Refl.

We can use the increment function to define a simple sequential sum function:

$$
\begin{aligned}
& \text { sum }: \text { forall }\{n n s p\} \rightarrow \text { Loc } n n s \rightarrow \text { Loc } 1 n s \rightarrow D I O() n s p n s \\
& \text { sum lout }=\text { ateach } l(\lambda i \rightarrow \text { readArrayl } i \gg \lambda n \\
& \quad \rightarrow \text { at }(\text { distr out } F z)(\text { increment out Fz } n \text { Refl }))
\end{aligned}
$$

The sum function takes an array as its argument, together with a reference to a single-celled array, out. It reads every element of the array, and increments out accordingly.

Finally, we can use both these functions to define a parallel sum:

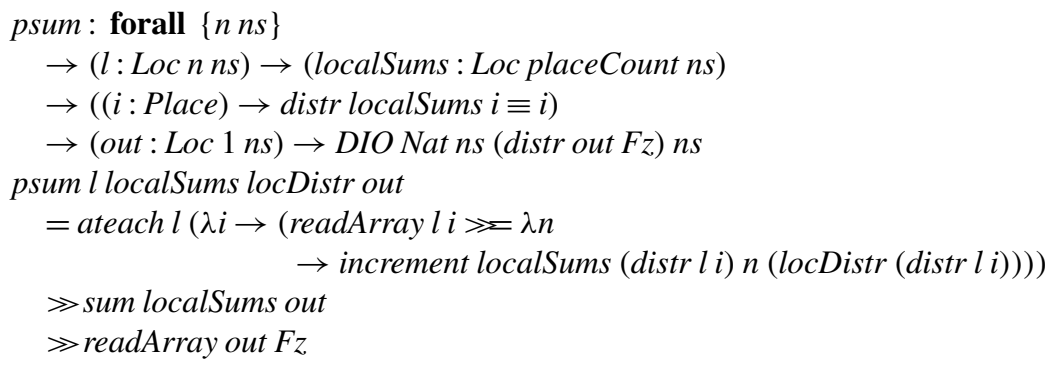

The psum function takes four arguments: the array $l$ whose elements you would like to sum; an array localSums that will store the intermediate sums; an assumption regarding the distribution of this array; and finally, the single-celled array to which we write the result. 
For every index $i$ of the array $l$, we read the value stored at index $i$, and increment the corresponding local sum. We then add together the local sums using our previous sequential sum function, and return the final result. We use our assumption about the distribution of the localSums array when calling the increment function. Without this assumption, we would have to use the place-shifting operation at to update a (potentially) non-local array index.

There are several interesting issues that these examples highlight. First of all, as our at function only works on computations returning a unit type, the results of intermediate computations must be collected in intermediate arrays.

More importantly, however, whenever we want to rely on properties of the global distribution, we need to make explicit assumptions in the form of proof arguments. This is rather unfortunate: it would be interesting to research how a specific distribution can be associated with an array when it is created. This would hopefully allow for a more fine-grained treatment of distributions and eliminate the need for explicit proof arguments.

\subsection{Defining distributions}

Throughout this section we have assumed the existence of a distribution, specifying how an array is distributed over the available places. Several built-in distributions are provided by $\mathrm{X} 10$, some of which we will now define. For the moment, we focus on defining a distribution of a single array, that is, functions of type Fin $n \rightarrow$ Place.

There are two atomic distributions: the constant distribution maps all the points of an array to a single place; the unique distribution maps the $i$-th index of an array to the $i$-th place.

$$
\begin{aligned}
& \text { constDistr }:\{n: \text { Nat }\} \rightarrow \text { Place } \rightarrow \text { Point } n \rightarrow \text { Place } \\
& \text { constDistr } p=\lambda i \rightarrow p \\
& \text { unique }: \text { Point placeCount } \rightarrow \text { Place } \\
& \text { unique } i=i
\end{aligned}
$$

We can compose any two such distributions, to form a larger distribution:

$$
\begin{aligned}
& \text { compose }: \text { forall }\{n m\} \\
& \quad \rightarrow(\text { Point } n \rightarrow \text { Place }) \rightarrow(\text { Point } m \rightarrow \text { Place }) \rightarrow(\text { Point }(n+m) \rightarrow \text { Place })
\end{aligned}
$$

Although the definition of compose is a bit tricky, there is a particularly elegant definition in the literature using views [2]. Of course, we can iterate the compose operator:

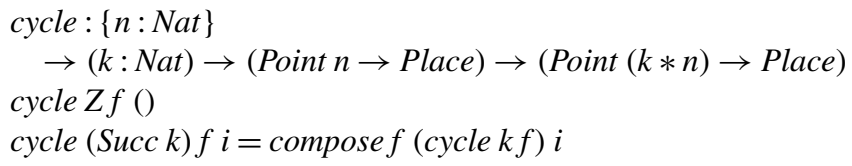

In the case where $k$ is equal to Zero, we need to define a function of type Fin Zero $\rightarrow$ Place. As Fin Zero has no inhabitants, this function will never be applied and we may omit the definition accordingly.

Using similar combinators, we can define distributions over all arrays, exploiting the obvious similarity between Loc and Fin. There are several other distributions supported by $\mathrm{X} 10$, that can be implemented along the same lines. 


\section{Discussion}

Using a dependently-typed host language, we have seen how to implement a domain-specific library for distributed arrays, together with an embedded type system that guarantees all array access operations are both safe and local. In contrast to existing work [10], there is no specific set of type rules; instead, equivalent properties are enforced by a general-purpose language with dependent types. We have defined semantics for our library in the form of a total, functional specification. Although this semantics may not take the form of deduction rules, they are no less precise or concise. Besides these functional specifications are both executable and amenable to computer-aided formal verification. More generally, this approach can be extended to other domains: a dependently-typed language accommodates domain specific libraries with their own embedded type systems [18].

Having said this, there are clearly several serious limitations of this work as it stands. First and foremost, I have assumed that every array only stores natural numbers, disallowing more complex structures such as multi-dimensional arrays. This can be easily fixed by defining a more elaborate Shape data type. In its most general form, the Shape data type could be defined as a list of types; a heap then corresponds to a list of values of the right type. I have decided to impose this restriction for the purpose of presentation. I believe that there is no fundamental obstacle preventing us from incorporating the rich region calculus offered by $\mathrm{X} 10$ in the same fashion.

Furthermore, the pure model is rather naive. It would be interesting to explore a more refined model, where every place maintains its own heap. As our example in the previous section illustrated, assuming the presence of a global distribution does not scale well. Decorating every array with a distribution upon its creation should help provide localityinformation when it is needed.

We have not discussed how code in the $I O$ or $D I O$ monad is actually compiled. At the moment, Agda can only be compiled to Haskell. Agda does provide several pragmas to customise how Agda functions are translated to their Haskell counterparts. The ongoing effort to support data parallelism in Haskell $[4,5]$ may therefore provide us with a most welcome foothold.

The domain-specific language for manipulating arrays presented in this paper contains an operation for the allocation of memory, but no operation for memory deallocation. The system presented here assumes garbage collection is handled automatically. This is not a fundamental limitation of this approach: in principle, dependently typed programming languages can enforce any invariant that can be described in constructive predicate logic. In particular, we could extend the shape data type not only capture the amount of data that has been allocated, but also record the locations that are still valid. Any memory access operation then needs to provide a proof that the location being accessed has not been deallocated. Where this is certainly feasible, there is still a substantial challenge in engineering a solution that does not put a strain on the programmer to provide explicit proofs or clutter type signatures with too much information.

There are many features of X10 that have not been discussed here at all. Most notably, I have refrained from modelling many of X10's constructs that enable asynchronous communication between locations, even though I would like to do so in the future.

Finally, it is necessary to explore larger examples to acquire a better understanding of how this approach scales. At the moment, I cannot predict how efficient the resulting code will be; nor do I know how difficult it will be to reason about large, realistic distributed algorithms. 
Acknowledgements A previous version of this article was published at the Symposium on Trends in Functional Programming in 2008. Thorsten Altenkirch, my PhD supervisor, suggested this line of research and helped prepare our original submission, for which I am grateful. I would also like to thank the anonymous reviewers for their valuable comments on both versions of this paper.

Open Access This article is distributed under the terms of the Creative Commons Attribution Noncommercial License which permits any noncommercial use, distribution, and reproduction in any medium, provided the original author(s) and source are credited.

\section{References}

1. Allen, E., Chase, D., Luchangco, V., Maessen, J.-W., Ryu, S., Steele, G.L. Jr., Tobin-Hochstadt, S.: The Fortress language specification. Technical report, Sun Microsystems, Inc. (2005)

2. Altenkirch, T., McBride, C., Morris, P.: Generic programming with dependent types. In: Backhouse, R., Gibbons, J., Hinze, R., Jeuring, J. (eds.) Spring School on Datatype-Generic Programming. LNCS, vol. 4719. Springer, Berlin (2007)

3. Atkey, R.: Parameterised notions of computation. In: Proceedings of the Workshop on Mathematically Structured Functional Programming (2006)

4. Chakravarty, M.M.T., Keller, G., Lechtchinsky, R., Pfannenstiel, W.: Nepal-nested data-parallelism in Haskell. In: Euro-Par 2001: Parallel Processing, 7th International Euro-Par Conference. LNCS, vol. 2150 (2001)

5. Chakravarty, M.M.T., Leshchinskiy, R., Jones, S.P., Keller, G., Marlow, S.: Data parallel Haskell: a status report. In: Proceedings of the 2007 Workshop on Declarative Aspects of Multicore Programming (2007)

6. Chamberlain, B.L., Choi, S.-E., Lewis, E.C., Lin, C., Snyder, L., Weathersby, D.: ZPL: a machine independent programming language for parallel computers. Softw. Eng. 26(3) (2000)

7. Chamberlain, B., Deitz, S., Hribar, M.B., Wong, W.: Chapel. Technical report, Cray Inc. (2005)

8. Charles, P., Grothoff, C., Saraswat, V., Donawa, C., Kielstra, A., Ebcioglu, K., von Praun, C., Sarkar, V.: X10: an object-oriented approach to non-uniform cluster computing. In: OOPSLA '05 (2005)

9. Grothoff, C., Palsberg, J., Saraswat, V.: Safe arrays via regions and dependent types. doi:10.1.1.98.8038

10. Grothoff, C., Palsberg, J., Saraswat, V.: A type system for distributed arrays. Unpublished draft

11. Hudak, P.: Building domain-specific embedded languages. ACM Comput. Surv. 28 (1996)

12. Liblit, B., Aiken, A.: Type systems for distributed data structures. In: POPL '00: Proceedings of the 27th ACM SIGPLAN-SIGACT Symposium on Principles of Programming Languages, pp. 199-213 (2000)

13. Löh, A.: lhs2TeX. http://people.cs.uu.nl/andres/lhs2tex/

14. McKinna, J., Wright, J.: A type-correct, stack-safe, provably correct, expression compiler in Epigram. J. Funct. Program. doi:10.1.1.105.4086

15. Nordström, B., Petersson, K., Smith, J.M.: Programming in Martin-Löf's Type Theory: An Introduction. Oxford University Press, London (1990)

16. Norell, U.: Towards a practical programming language based on dependent type theory. $\mathrm{PhD}$ thesis, Chalmers University of Technology (2007)

17. Nystrom, N., Saraswat, V., Palsberg, J., Grothoff, C.: Constrained types for object-oriented languages. In: OOPSLA '08: Proceedings of the 23rd ACM SIGPLAN Conference on Object-Oriented Programming Systems Languages and Applications, pp. 457-474 (2008)

18. Oury, N., Swierstra, W.: The power of Pi. In: ICFP '08: Proceedings of the 13th ACM SIGPLAN International Conference on Functional Programming (2008)

19. Jones, S.P. (ed.): Haskell 98 Language and Libraries—The Revised Report. Cambridge University Press, Cambridge (2003)

20. Jones, S.P., Vytiniotis, D., Weirich, S., Washburn, G.: Simple unification-based type inference for GADTs. In: ICFP '06: Proceedings of the 11th ACM SIGPLAN International Conference on Functional Programming (2006)

21. Scholz, S.-B.: Single assignment C-efficient support for high-level array operations in a functional setting. J. Funct. Program. 13(6), 1005-1059 (2003)

22. Swierstra, W.: A functional specification of effects. PhD thesis, University of Nottingham (2009)

23. Swierstra, W., Altenkirch, T.: Beauty in the beast: a functional semantics of the awkward squad. In: Proceedings of the ACM SIGPLAN Haskell Workshop (2007) 